\title{
AC 2010-2078: ASSISTIVE ROBOTICS COMPETITION ROBOWAITER: A NEW APPROACH TO SOCIALLY RESPONSIBLE ROBOTICS EDUCATION
}

\section{David Ahlgren, Trinity College}

David J. Ahlgren is Karl W. Hallden Professor of Engineering at Trinity College and is Director and Host of the Trinity College Fire-Fighting Home Robot Contest. Professor Ahlgren has been a faculty member at Trinity College since 1973. His current professional interests include educational robotics with real-world applications. Prof. Ahlgren received the B.S. in Engineering from Trinity College, the M.S. in Electrical Engineering from Tulane University, and the Ph.D. in Electrical Engineering from The University of Michigan, Ann Arbor.

\section{Igor Verner, Technion-Israel Institute of Technology}

Igor M. Verner is Associate Professor at the Department of Education in Technology and Science, Technion - Israel Institute of Technology. He received the M.S. degree in Mathematics from the Urals State University and the Ph.D. in computer aided design systems in manufacturing from the Urals State Technical University (1981), Yekaterinburg, Russia. Dr. Verner is a certified teacher of mathematics and technology in Israel. His research interests include experiential learning, cognitive and affective development, design projects, and robotics. He is involved in organization of international robot competitions and guidance of school teams. 


\title{
Assistive Robotics Competition RoboWaiter: A New Approach to Integrating Robotics and Socially Responsible Education
}

\begin{abstract}
This paper reports on RoboWaiter-the first robot competition in the rapidly growing area of assistive robotics - that was conducted in conjunction with the annual international Trinity College Fire-Fighting Home Robot Contest in 2009. Organized with active participation of members of the Connecticut Council on Developmental Disabilities, RoboWaiter's overarching goals were to promote awareness of the needs of persons with disabilities while providing an engineering challenge to designers of all levels of skill within an atmosphere of friendly, teambased competition. An additional goal was to encourage students to think about responsibilities of engineers to society. In this paper we describe the RoboWaiter 2009 assignment and the associated engineering challenge, and we present results of our educational studies. We also discuss the potential of the RoboWaiter competition for developing and demonstrating assistive robotics technologies, providing a fruitful environment for robotics and opportunities for socially responsible education.
\end{abstract}

\section{Need for Assistance}

According to the Bureau of Industry and Security, U.S. Department of Commerce, more than $17 \%$ of Americans have a disability, and half of that cohort has a severe disability. The number of persons with severe disabilities is increasing and will continue to grow as the population ages [1]. Many persons with disabilities benefit from an assistive technology device, an "item, piece of equipment, product or system, whether acquired commercially off the shelf, modified, or customized, that is used to increase, maintain, or improve the functional capabilities of persons with disabilities. "[2]. In a 2005 survey by the U.S. Department of Education, National Institute on Disability and Rehabilitation Research, $64 \%$ of respondents used some assistive technology. The most frequently used were devices that enhance mobility (canes, crutches, walkers, scooters and wheelchairs), hearing aids, and oxygen tanks. Most respondents who used AT said it made them more productive and more aware of their rights [3]. The U.S. Bureau of Commerce report points out that there is an active, and rapidly growing, assistive technology industry that manufactures more than 17,000 products, and employed over 20,000 workers [1]. Still, according to the Assistive Technology Industry Association, the number of people currently using assistive technology is only a fraction of those who could benefit from it [2]. Thus it is appropriate to raise awareness among engineering students about the needs of those people and to encourage them to solve associated design problems.

Recent attention has focused on the use of robots as assistive agents. Such robots help people with disabilities through physical or social interaction and include rehabilitation robots, wheelchair robots and other mobility aides, companion robots, manipulator arms, and educational robots. An example of an assistive robot is a robot at Georgia Tech that helps persons with disabilities to carry out everyday activities [4]. The robot can fetch objects and open 
drawers on command, replacing functions carried out by service dogs. To interact with the Georgia Tech service robot, a person with disabilities points a laser at an object and gives a verbal command to the robot; an example is a command to open a drawer or to close a door [4].

\section{Genesis of an Assistive Robotics Competition}

The need for robotic devices to help persons with disabilities encouraged development of an assistive robot event consistent with a continuing effort to upgrade and enhance the Trinity College Fire-Fighting Home Robot Contest (TCFFHRC). Drawing 120 teams each year from around the world, the TFFFHRC has been held in Hartford, CT since 1994 [5, 6]. The Connecticut Council on Developmental Disabilities (CTCDD) has sponsored the assistive robotics event since 2007. The first event, "Find the Child" took place as part of the 2007 and 2008 fire-fighting contests. In "Find the Child", autonomous robots searched for a warm doll representing an autistic child hiding from a perceived threat. Feedback from participants, CTCDD members, and supporters, collected by means of questionnaires and interviews, suggested revising the event to include a more relevant and challenging assistive robotics theme.

There followed a planning effort by a group comprising three members of the Connecticut Council on Developmental Disabilities and the lead author. The group met on several occasions during the summer and fall of 2008 to explore possible topics for the contest. Eventually the group settled on a theme that represented a real area of concern to Council members - the need for a person with disabilities to obtain food from a refrigerator during an emergency situation when a personal assistant is not able to be present. Thus was born the idea of an autonomous robot that would serve as a server or waiter and a unique contest, RoboWaiter, the only assistive robot competition in 2009-2010 [7].

The TCFFHRC and the RoboWaiter contest that emerged from the planning process are compatible, mutually supportive, and strongly coupled events. They share many goals- to stimulate creativity and to encourage students of all ages to engage a project that has societal benefits, for example - and both encourage development of new technologies. Both take place in scale-model arenas outfitted to the contest theme, and both require participants to solve engineering design problems spanning several disciplines. The scoring equations for both events emphasize reliability over speed. Persons who enter either competition have the opportunity to participate in other events on the contest weekend - a theoretical test, a robotics symposium, and a poster session, for example. Finally, the RoboWaiter and fire-fighting contests present difficult engineering challenges involved with integrating such diverse design elements as algorithm development, software design, gripper design, sensing and obstacle avoidance, and motor control.

\section{The 2009 RoboWaiter Challenge}

The planning group decided that the event would take place in a scale model kitchen that included typical kitchen fixtures. It was convenient for the kitchen to measure $2.5 \mathrm{~m} \times 2.5 \mathrm{~m}$, the same size as the Trinity fire-fighting contest arenas. The RoboWaiter rules allow each robot to have three runs. Once started by the contest judge, the robot must navigate autonomously from its home position to a scale-model refrigerator, pick up a plate of food from a shelf, navigate to 
the table where a person with mobility impairment is sitting, places the plate on it, and return to the home position. Robots must avoid collisions with obstacles - a sink, a chair, and a second, elderly person-whose positions are not precisely known (Figures 1, A and B). A beacon, consisting of three bright red light-emitting diodes (LEDs) mounted on edge of the shelf, indicates the location of the plate. The plate is a plastic pet food can top filled with Cheerios. The scoring formula weighs reliability first (the top group consists of robots that succeed on all three runs) and uses time as a differentiator within each reliability group.

Ten teams entered RoboWaiter 2009, and Connecticut Council on Developmental Disabilities members served as judges. The winning robot "James the Bot" was built as a term project by a team of freshman engineering students from Grand Valley State University in Michigan [8]. James the Bot succeeded on all three runs. A professional engineer from Florida built the secondrobot, and a high school student from Ort Givat Ram Jerusalem developed the third place robot "Tsunami" (Figure 3). The geographical and age diversity of these teams illustrates the wide interest spawned by this event.

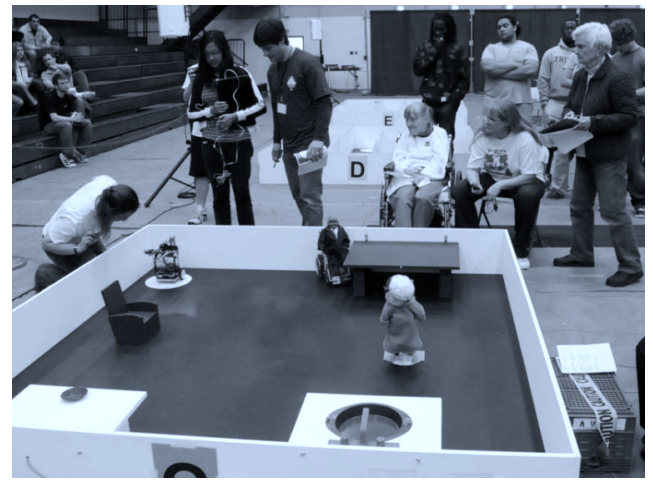

Figure 1: A. 2009 RoboWaiter arena

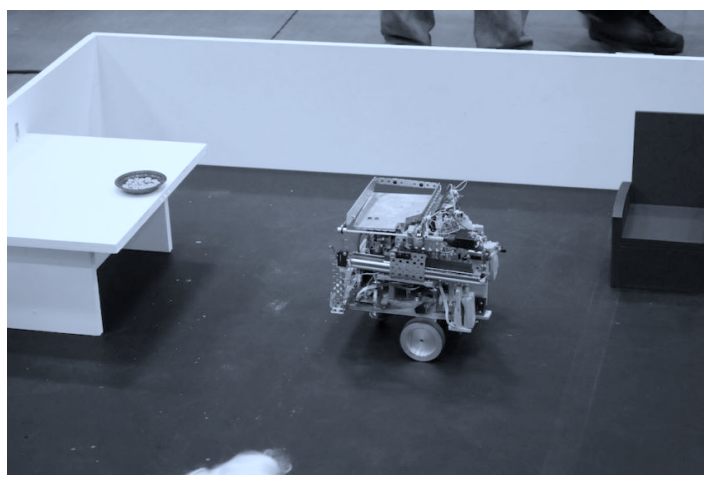

B. One of the robots approaching plate

\section{Involvement of People with Disabilities in the RoboWaiter Competition}

Traditional participants of robot contests are contestants - students, hobbyists, and engineerswho design, demonstrate, and compete with their robots. In case of the RoboWaiter an additional category of participants is included - people connected to the Connecticut Council of Developmental Disabilities (CCDD). For these participants the RoboWaiter contest opens an opportunity of active social interaction in a public event where they can learn about modern assistive technology and personally engage in discussions with engineers on developing new assistive robots to answer their needs.

Persons with sensory and motor impairments represent a serious challenge for education and care programs. Their limited range of movements and minimal interaction with the environment can prevent the acquisition of adaptive skills and increased risk of a poor quality of life. Recently, considerable emphasis has been placed on finding ways of counteracting the aforementioned risks. Researchers have begun to develop intervention programs, which involve the presentation of favorite stimuli across various periods of the day in an attempt to increase indices of happiness with these people [9]. 
RoboWaiter opens for this category of participants various opportunities for joyful experiences, both in participating in the contest organization and in attending and working at the contest itself. Organizational activities include the following: developing the new contest theme and formulating contest rules, formulating contest judgment criteria, recruiting participants, fundraising, public relations, and recommending improvements. At the contest the joyful experiences include observing the action, judging the competition, providing feedback for team projects and contest organizers, learning about robotics, participating in the contest symposium, encouraging students and teachers, testing and evaluating new assistive robots, and giving prizes.

\section{Evaluation}

The goal of our evaluation was to measure the impact of the 2009 competition and to see if RoboWaiter met its goals. Our special interest was to get evidence on to what extent the contestants are motivated by the engineering, humane and social challenges of the assistive robotics competition.

Here, the term "humane challenge" expresses the educational challenge of teaching robotics in the humanistic cultural context. By involving students in assistive robotics projects we try to foster consideration for real needs of people with disabilities, to evoke feelings of compassion and sympathy to them. The term "social challenge" concerns fostering students' awareness about social problems of people with disabilities and thinking on how assistive robotics can really help in solving these problems.

We collected information by our observations and through an educational survey given immediately after the contest; the survey form is presented in the Appendix. The survey focused on testing perceptions and attitudes of two target groups: (1) those who developed RoboWaiter robots, and (2) supporters, persons with disabilities or their families who attended the contest, served as volunteers, or participated in developing the contest.

\section{Educational Survey}

As one part of the educational survey, each participant was given a list of reasons for competing and asked to rate the importance of each using the scale from 1 (not important) to 5 (very important). A similar survey section was given to contestants and the supporters, but the lists of possible reasons for their involvement were different.

The answers from the survey groups are shown in Tables I and II. As found, all listed reasons were relevant. The highest evaluations among the contestants were for the engineering and humane challenges and curiosity about the new competition. 
Table I: Possible reasons for participation in RoboWaiter (Contestants, N=15)

\begin{tabular}{|l|c|c|}
\hline \multicolumn{1}{|c|}{ Possible Reason } & Avg. & Std. Dev. \\
\hline 1. Engineering challenge & 4.54 & 1.02 \\
\hline 2. Humane challenge & 3.93 & 1.20 \\
\hline 3. Social challenge & 3.31 & 1.12 \\
\hline 4. Interest in assistive robotics & 3.57 & 1.06 \\
\hline 5. Curiosity & 3.99 & 1.16 \\
\hline 6. Interest to major in the subject & 3.53 & 1.29 \\
\hline 7. Job, scholarship or advanced studies opportunities & 3.40 & 1.18 \\
\hline
\end{tabular}

Evaluations given by the supporters were high for all mentioned reasons, but the highest grades were given to attracting attention to needs of users of assistive technology and increasing awareness among its potential users.

Table II: Possible reasons for your support for RoboWaiter (Supporters, $\mathrm{N}=4$ )

\begin{tabular}{|l|c|c|}
\hline \multicolumn{1}{|c|}{ Possible Reason } & Avg. & Std. Dev. \\
\hline 1. Interest in robotics & 4.00 & 1.41 \\
\hline 2. To foster development of assistive technology & 3.75 & 1.50 \\
\hline 3. To attract attention to needs of users of assistive technology & 4.50 & 1.00 \\
\hline 4. To increase awareness among potential users of assistive technology & 4.25 & 0.96 \\
\hline 5. To inspire dialogue between users and developers of assistive technology & 4.00 & 0.82 \\
\hline 6. To introduce students to the assistive technology subject & 4.25 & 0.96 \\
\hline
\end{tabular}

\section{Written Interviews}

Along with colleagues from the CTCCD the authors presented an invited workshop "RoboWaiter: The Assistive Mobile Robot Competition" at the 2009 Annual Conference of the National Association of Councils on Developmental Disabilities (NACDD), Albuquerque, NM [10]. The workshop gave us incentive more deeply to analyze perceptions and attitudes of the attendees. For this purpose, prior to the workshop and four months after the competition we conducted a series of written interviews with a number of contestants and supporters. We find the responses very instructive; the most interesting comments are presented below.

A (Professional engineer from Florida, participant):

I had not given assistive robotics much thought before the competition. As an engineer, I tend to get focused on a task and forget about people. The project gave me lots of new ideas. I think it was a very effective way to get people thinking about what robotic technology can do for people with disabilities.

Socially responsible education is very important. Students have to get out in the real world. For an assistive robotics project, I think it would be very valuable for students to interact with people who have disabilities and get their feedback. 
B (University student from Connecticut, participant):

In RoboWaiter learning robotics and programming, as well as social, moral, and humane issues take place. These experiences help students realize what their education is leading up to, and how they can end up changing the lives of so many people.

It was great to have participation of people with disabilities. There is no downside to them being there and seeing how robots could potentially help. Their participation can also help in coming up with new ideas, as they converse with the builders.

C (High school student from Israel, participant):

RoboWaiter made me more conscious of the main purpose of building a robot--to help people. I saw people with disabilities enjoying the contest and was happy that we made them to have a smile on their face when they saw the robot work. This was more important than the result score.

More people should come to compete and more people with disabilities should come to view the competition because it make them happy when they see the robots and people who try to help them with technical equipment and give them hope.

D (Member of CCDD, disabled):

I would like to learn more about how robots can assist people with disabilities in various places such as in homes, cars, employment, etc.

I feel RoboWaiter is more of education and an awakening to the participants that robots could be essential to people with disabilities of any age...more teams should have the opportunity to come to the competitions because RoboWaiter is a wonderful experience.

E (Member of CCDD, disabled):

I have watched the robotic contests at Trinity College. I've enjoyed them. They are exciting!! I think the RoboWaiter gives a realistic demonstration of a service that a robot could provide to someone with a disability.

The RoboWaiter competition teaches students that, through technology, they can help people with disabilities in their own homes. Engineering students come to understand that with increasingly sophisticated devices being developed and produced, persons with disabilities will enjoy happier, more productive lives.

In summary, the interviews point to appreciation of the RoboWaiter by contestants and persons with disabilities.

Real-time Survey at the NACDD Conference

The goal of our NACDD workshop was to present and promote the assistive robotics competition RoboWaiter before members of Councils of Developmental Disabilities. Creating effective dialogue with the audience during the session was difficult but crucial for achieving the goal. We solved this challenging communication problem by using Personal Response Systems (PRS) or clickers. For attendees of our talk this was absolutely new technology, but they found it 
simple and convenient. For us, the use of PRS gave an opportunity to operatively collect data on perceptions and attitudes of the target group. Some of the data are presented below.

Among 18 session attendees there were council officers, people with disabilities, and researchers. We asked the attendees to what extent they agreed or disagreed with the following statements:

1. In the near future robots will be in wide use in helping people with disabilities.

2. People need basic knowledge in robotics in order to use home robots properly.

3. Engineering education should foster students' awareness of the needs of people with disabilities.

4. Educational programs in universities and schools should include practice directed to help people.

5. People with disabilities can benefit from active participation in assistive robotics programs.

6. Collaboration between Councils of Developmental Disabilities and universities offering assistive robotics education is recommended.

Responses of the attendees are summarized in Table III

Table III: Summary of Results by Question (\%)

\begin{tabular}{|l|c|c|c|c|c|c|}
\hline \multicolumn{1}{|c|}{ Question: } & 1 & 2 & 3 & 4 & 5 & 6 \\
\hline Strongly Agree & 71 & 12 & 59 & 76 & 94 & 81 \\
\hline Agree & 18 & 29 & 24 & 24 & 6 & 19 \\
\hline Neither agree nor disagree & 0 & 18 & 12 & 0 & 0 & 0 \\
\hline Disagree & 12 & 18 & 6 & 0 & 0 & 0 \\
\hline Strongly Disagree & 0 & 24 & 0 & 0 & 0 & 0 \\
\hline
\end{tabular}

As the issue of assistive robotics was new for many attendees, it was difficult for us to hypothesize their reactions. We were surprised to find that in many respects the attendees shared our opinions. All the attendees believe that educational programs in universities and schools should include practice directed to help people, that people with disabilities can benefit from active participation in assistive robotics events, and that collaboration between Councils of Developmental Disabilities and universities in the area of assistive robotics education can be recommended. Also, the majority of the attendees agreed that in the near future assistive robots will be in wide use and that engineering education should foster students' awareness of the needs of people with disabilities. Responses to the statement that "people need basic knowledge in robotics in order to use home robots properly" were varied. While part of the attendees agreed with the statement, the other part disagreed, saying that assistive technology should be developed so that unskilled users could manage it. 


\section{Conclusion}

In this paper we described the new RoboWaiter competition, discussed briefly the engineering challenge, and presented the results of participant and supporter surveys. The first-year success of RoboWaiter opens many possibilities for future competitions. For example, in 2010 the contest will offer a new RoboWaiter Advanced Division that will require robots to interact with a "smart" refrigerator and to retrieve plates of food from two different shelves. In the new division, an encoded start signal will bear information about where the food is located. A sensor embedded in the kitchen floor will enable the robot to open the refrigerator door, and a modulated IR beam emitted by the refrigerator will guide the robot to the plate. These new options lend greater realism and add "smart home" features to the contest.

Based on our surveys, we find that RoboWaiter presented a significant engineering challenge and that designers were eager to take on the new work and to test their robots in the competition. Curiosity was also an important motivator, but equally so was the humane challenge. Participants recognized that their work might have importance beyond pure technical achievement; such factors as social challenge, interest in assistive robotics, interest in majoring in the subject, and job and scholarship or advanced studies opportunities were also strong motivators. Among the sample of supporters surveyed, primary motivators were associated with attracting attention to the needs of persons with disabilities, creating awareness, introducing students to the assistive technology subject, and creating dialogue among users and developers of assistive technology. Supporters were very interested in robotics, consistent with their involvement in the contest and their continuing support for it.

While engaging the participants, the contest involved real persons with disabilities in developing an international robotics event and participating in it as judges and volunteers. Feedback from these individuals indicated that robot competitions might play an important social role in drawing public attention to the need for new technologies, inspiring technological development, and fostering engineering education. We conclude that RoboWaiter forged new links between supporters and designers that can lead to fruitful interactions in the future.

\section{Acknowledgements}

The authors acknowledge the generous support of the Connecticut Council on Developmental Disabilities and give special thanks to Ed Preneta, Mary-Ann Langton, Joyce Baker, and Armand Legault for their dedication to the RoboWaiter project. We acknowledge also the many contributions of Trinity staff members Jessica Studinski and Andrew Musulin.

\section{References}

1. U.S. Department of Commerce Technology Assessment of the U.S. Assistive Technology Industry, http://www.bis.doc.gov/defenseindustrialbaseprograms/osies/defmarketresearchrpts/assisttechrept/4intro.htm 2. Assistive Technology Outcomes and Benefits (2004). Assistive Technology Industry Association and Special Education Assistive Technology Center, http://www.atia.org/i4a/pages/index.cfm?pageID=3681 . 3. Assistive Technology and Information Technology Use and Need by Persons with Disabilities in the United States (2005). U.S. Department of Education National Institute on Disability and Rehabilitation Research, http://www.ed.gov/rschstat/research/pubs/at-use/at-use-2001.pdf. 
4. Hai Nguyen and Charles C. Kemp. "Bio-inspired Assistive Robotics: Service Dogs as a Model for Human-Robot Interaction and Mobile Manipulation." In Proceedings of the IEEE RAS/EMBS International Conference on Biomedical Robotics and Biomechatronics (BioRob 2008), pp. $542\{549$ (2008).

5. I. M. Verner, and D. J. Ahlgren. "Robot Projects and Competitions as Education Design Experiments." Intelligent Automation and Soft Computing Journal, Special Issue on Educational Robotics, 13, 1, 2007, pp. 57-68.

6. Trinity College Fire-Fighting Home Robot Contest website: www.trincoll.edu/events/robot.

7. According to www.robots.net, 119 different robot competitions will be held in 2009-2010 academic/school year. Among them there is only one assistive robotics competition: RoboWaiter.

8. http://www.mlive.com/news/grand-rapids/index.ssf/2009/04/grand_valley_state_university_21.html, http://www.gvsu.edu/gvnow/?articleId=A17D7211-F926-D81C-49B7074B8C08859 E

9. Lancioni, G. E., O'Reilly, M. F., Campodonico, F., and Mantini, M. (2002). Increasing Indices of Happiness and Positive Engagement in Persons With Profound Multiple Disabilities, Journal of Developmental and Physical Disabilities, 14(3), 231-237.

10. D. Ahlgren, I. Verner, M. Langton J. Baker, A. Legault, E. Preneta. "RoboWaiter: The Assistive Mobile Robot Competition". Workshop presented at the National Association of Councils on Developmental Disabilities Annual Conference, Albuquerque, October, 2009. 


\section{Appendix: 2009 RoboWaiter Survey}

\section{TRINITY COLLEGE FIRE FIGHTING HOME ROBOT CONTEST \\ RoboWaiter - Assistive Robotics Competition Survey '09}

\section{General}

State / Country

Your Name

\begin{tabular}{ll} 
& State / Country \\
\hline RoboWaiter: __ Contestant __ Supporter
\end{tabular}

Involvement in RoboWaiter:

Contestant

Contest division(s)

Contestant details: Robot name(s)

Professional or student position

Institution

Curricular __ Extracurricular

Form of your participation in the RoboWaiter:

Supporter details: _ Individual __ Organization member (specify)

Forms of support: Current Future

Your experience with assistive technology \& assistive robotics

2. Goals

Please present your view of the goals of the assistive robotics competition

\section{Strategies}

Please reflect on matching of the assistive robotics competition goals by the current RoboWaiter contest. Suggest strategies that can improve the RoboWaiter.

\section{Reasons}

The first of the tables below is intended for contestants and the second (reverse side) for supporters. Please answer in the proper table. The tables are similar: the first column includes a list of possible reasons for participation in or support of the RoboWaiter. In the second column please estimate the level of importance of each of the reasons for your personal involvement in the contest, using the scale from 1 - not important to 5 - very important.

\begin{tabular}{|l|l|}
\hline \multicolumn{1}{|c|}{ Possible reasons for participation in the RoboWaiter } & Level of importance \\
\hline 1. Engineering challenge & \\
\hline 2. Humane challenge & \\
\hline 3. Social challenge & \\
\hline 4. Interest in assistive robotics & \\
\hline 5. Curiosity & \\
\hline 6. Interest to major in the subject & \\
\hline 7. Job, scholarship or advanced studies opportunities & \\
\hline Other reasons (specify): & \\
\hline & \\
\hline & \\
\hline
\end{tabular}




\section{Contestants:}

\begin{tabular}{|l|l|}
\hline \multicolumn{1}{|c|}{ Possible reasons for your support of the RoboWaiter } & Level of importance \\
\hline 1. Interest in robotics & \\
\hline 2. To foster development of assistive technology & \\
\hline 3. To attract attention to needs of users of assistive technology & \\
\hline $\begin{array}{l}\text { 4. To increase awareness among potential users of assistive } \\
\text { technology }\end{array}$ & \\
\hline $\begin{array}{l}\text { 5. To inspire dialogue between users and developers of } \\
\text { assistive technology }\end{array}$ & \\
\hline 6. To introduce students to the assistive technology subject & \\
\hline & \\
\hline Other reasons (specify): & \\
\hline & \\
\hline
\end{tabular}

Supporters:

\begin{tabular}{|l|l|}
\hline \multicolumn{1}{|c|}{ Possible reasons for your support of the RoboWaiter } & Level of importance \\
\hline 1. Interest in robotics & \\
\hline 2. To foster development of assistive technology & \\
\hline 3. To attract attention to needs of users of assistive technology & \\
\hline $\begin{array}{l}\text { 4. To increase awareness among potential users of assistive } \\
\text { technology }\end{array}$ & \\
\hline $\begin{array}{l}\text { 5. To inspire dialogue between users and developers of } \\
\text { assistive technology }\end{array}$ & \\
\hline 6. To introduce students to the assistive technology subject & \\
\hline & \\
\hline Other reasons (specify): & \\
\hline & \\
\hline
\end{tabular}

\section{Specific features}

From your observations, please reflect on specific features of the RoboWaiter in comparison with other competition categories

\section{Popularization}

Please share your ideas about possible ways for popularization of assistive robotics and the RoboWaiter

\section{Activism}

Please share your ideas on how to facilitate participation of disabled people in the RoboWaiter as contestants. 
The Authors:

David J. Ahlgren is Karl W. Hallden Professor of Engineering at Trinity College and is Director and Host of the Trinity College Fire-Fighting Home Robot Contest. Professor Ahlgren has been a faculty member at Trinity College since 1973 . His current professional interests include educational robotics with real-world applications. Prof. Ahlgren received the B.S. in Engineering from Trinity College, the M.S. in Electrical Engineering from Tulane University, and the Ph.D. in Electrical Engineering from The University of Michigan, Ann Arbor.

Igor M. Verner is Associate Professor at the Department of Education in Technology and Science, Technion - Israel Institute of Technology. He received the M.S. degree in Mathematics from the Urals State University and the Ph.D. in computer aided design systems in manufacturing from the Urals State Technical University (1981), Yekaterinburg, Russia. Dr. Verner is a certified teacher of mathematics and technology in Israel. His research interests include experiential learning, cognitive and affective development, design projects, and robotics. He is involved in organization of international robot competitions and guidance of school teams. 